\title{
A triple-network organization for the mouse brain
}

Francesca Mandino (D) ${ }^{1,2,3,14}$, Roël M. Vrooman ${ }^{4,14}$, Heidi E. Foo ${ }^{1,5,14}$, Ling Yun Yeow ${ }^{1,14}$, Thomas A. W. Bolton (DD ${ }^{6}$, Piergiorgio Salvan7 Chai Lean Teoh ${ }^{1}$, Chun Yao Lee ${ }^{1}$, Antoine Beauchamp ${ }^{8}$, Sarah Luo ${ }^{1}$, Renzhe Bi (D) ${ }^{1}$, Jiayi Zhang ${ }^{1,9}$, Guan Hui Tricia Lim ${ }^{1,10}$, Nathaniel Low ${ }^{1}$, Jerome Sallet ${ }^{7,11}$, John Gigg (D) $^{2}$, Jason P. Lerch ${ }^{7,8}$, Rogier B. Mars $\mathbb{D}^{7,12}$, Malini Olivo ${ }^{1}$, Yu Fu ${ }^{1}$ and Joanes Grandjean (iD ${ }^{1,4,13 \bowtie}$

(c) The Author(s) 2021

The triple-network model of psychopathology is a framework to explain the functional and structural neuroimaging phenotypes of psychiatric and neurological disorders. It describes the interactions within and between three distributed networks: the salience, default-mode, and central executive networks. These have been associated with brain disorder traits in patients. Homologous networks have been proposed in animal models, but their integration into a triple-network organization has not yet been determined. Using resting-state datasets, we demonstrate conserved spatio-temporal properties between triple-network elements in human, macaque, and mouse. The model predictions were also shown to apply in a mouse model for depression. To validate spatial homologies, we developed a data-driven approach to convert mouse brain maps into human standard coordinates. Finally, using high-resolution viral tracers in the mouse, we refined an anatomical model for these networks and validated this using optogenetics in mice and tractography in humans. Unexpectedly, we find serotonin involvement within the salience rather than the default-mode network. Our results support the existence of a triple-network system in the mouse that shares properties with that of humans along several dimensions, including a disease condition. Finally, we demonstrate a method to humanize mouse brain networks that opens doors to fully data-driven trans-species comparisons.

Molecular Psychiatry (2022) 27:865-872; https://doi.org/10.1038/s41380-021-01298-5

\section{INTRODUCTION}

Brain disorders are defined by altered patterns of brain structure and function, which provide insights into their underlying mechanisms [1, 2]. The neuroimaging correlates for neuropsychiatric disorders have been shown to cluster into overlapping brain areas [3-5]. The triple-network model of psychopathologies proposes a theoretical framework based on the interactions within and between three networks to explain psychiatric and neurological traits [1]. The model encompasses three networks: the default-mode, salience, and central executive networks. These are defined by their characteristic spatial (overlapping areas) and temporal (e.g., default-mode and central executive networks' anticorrelation) properties [6-8].

These networks are not unique to the human brain. Putative homologous networks have also been reported in nonhuman primates and rodents [9-12]. Determination of the underlying pathological mechanisms often relies on animal models, where invasive manipulations and measurements reveal actionable targets for the development of new therapies. Hence, the characterization of these networks in animals allows for translatable insights applicable to humans. Between-species comparisons rely on spatial assumptions of equivalency, yet these remain non-trivial to determine [13]. Here, we seek to characterize network homologies using a data-driven approach. To forgo approximate matching between species, we developed a new method to convert brain maps using spatial transcriptomic similarity between the mouse and the human brain [14, 15]. We propose that homologous brain states exist in three mammalian species commonly used in neuroscience research, namely human, macaque and mouse. To validate this, we generated humanized synthetic brain states derived from mouse brain maps and spatially compared them to matched human brain states. Beyond spatial homology, we hypothesize that transitions between brain states are conserved between the species examined, thus, defining temporal similarity.

In this work, we establish shared spatio-temporal brain state properties between the human, macaque, and mouse brains. We test the predictions of the triple-model in mice by examining brain

\footnotetext{
${ }^{1}$ Singapore Bioimaging Consortium, Agency for Science, Technology and Research, 11 Biopolis Way, Singapore 138667, Singapore. ${ }^{2}$ Faculty of Biology, Medicine and Health, The

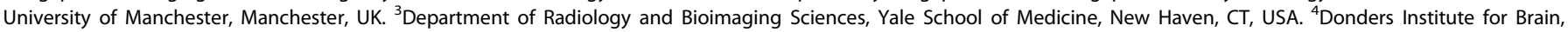
Cognition and Behaviour, Radboud University Medical Center, Nijmegen, The Netherlands. ${ }^{5}$ Centre for Healthy Brain Aging, CHeBA, School of Psychiatry, University of New South Wales Medicine, Kensington, Sydney, NSW 2052, Australia. ${ }^{6}$ Neurosurgery Service and Gamma Knife Center, Centre Hospitalier Universitaire Vaudois, Lausanne, Switzerland.

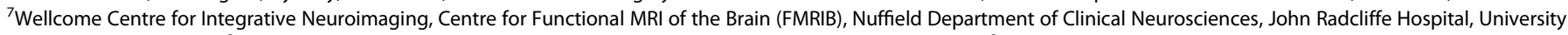
of Oxford, Oxford, UK. ${ }^{8}$ Department of Medical Biophysics, University of Toronto, Toronto, ON, Canada. ${ }^{9}$ Centre for Research and Development in Learning, Nanyang

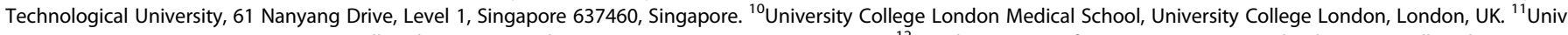
Lyon, Université Lyon 1, Inserm, Stem Cell and Brain Research Institute U1208, 69500 Bron, France. ${ }^{12}$ Donders Institute for Brain, Cognition and Behaviour, Radboud University

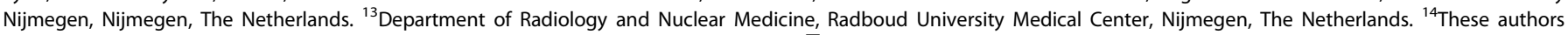
contributed equally: Francesca Mandino, Roël M. Vrooman, Heidi E. Foo, Ling Yun Yeow. ${ }^{\bowtie}$ email: joanes.grandjean@radboudumc.nl
}

Received: 18 May 2021 Revised: 21 August 2021 Accepted: 8 September 2021

Published online: 14 October 2021 
state occurrences in a dataset containing resting-state recordings in a mouse model for depression [16]. We then generate a precise neuroanatomical model for the triple-network organization using a high-resolution mouse tracer dataset. Finally, we validate this anatomical model using functional stimulation of two key nodes of the salience network commonly implicated with depressive disorders, namely the dorsal raphe (DR) nucleus and the insular area. Using a combination of optogenetic stimulation during behavior and whole-brain imaging, we identify interactions between elements of the salience and default-mode networks associated with positive valence and cross-reference this with a meta-analysis of the human neuroimaging literature. Taken together, we demonstrate that triple-network model predictions for depressive traits apply in the mouse. Last but not least, our neuroanatomical model suggests a revision of the monoamine contribution in the regulation of these networks.

\section{METHOD}

\section{Data and code availability}

The complete preprocessed mouse functional Magnetic Resonance Imaging (fMRI) datasets are freely available at https://doi.org/10.34973/1he1-5c70 (resting-state) and https://doi.org/10.34973/raa0-5z29 (optogenetics, CAPs). The S1200 human connectome dataset is available at https://db. humanconnectome.org/ [17]. The viral tracer data is available from https:// connectivity.brain-map.org/ [18]. The mouse template is available at https:// atlas.brain-map.org/. The complete code to reproduce the study is freely available as an $\mathrm{R}$ notebook at https://gitlab.socsci.ru.nl/preclinicalneuroimaging/insula. The repository also includes tables, figures, and imaging assets. The code to produce synthetic brain maps is available at https://gitlab. socsci.ru.nl/preclinical-neuroimaging/homologous-brain-expression-map.

\section{Data processing and analysis}

Mouse and macaque data were preprocessed as follows. Anatomical images were corrected for B1 field (N4BiasFieldCorrection), denoised (Denoiselmage), brain-masked (antsBrainExtraction.sh) and registered using SyN diffeomorphic estimation (antsRegistration) to the AIBS template (mouse, https://atlas.brain-map.org/) or F99 template (macaque) [19] using ANTs [20]. Functional images were despiked (3dDespike), motion-corrected (3dvolreg), corrected for B1 field, denoised, and brain-masked, before being registered linearly to their corresponding anatomical images. Resting-state fMRI (rsfMRI) data were band-pass filtered (3dBandpass, $0.01-0.25 \mathrm{~Hz}$ for the mouse, $0.01-0.10 \mathrm{~Hz}$ for the macaque). Optogenetic fMRI (ofMRI) data were high-pass filtered $(0.01 \mathrm{~Hz})$. Independent component analysis (melodic) was applied, and nuisance components were determined using FIX before being regressed out (fs/regfilt). Z-statistics for evoked activity (ofMRI) or functional connectivity (rsfMRI) were determined using fsl_glm. The code to preprocess the mouse and macaque datasets is available at https://github.com/grandjeanlab/MouseMRIPrep.

\section{Brain state analysis}

Brain states were derived using co-activation patterns (CAPs). These were estimated using the TbCAPs toolbox (https://c4science.ch/source/ CAP_Toolbox.git) [21]. The anesthetized mouse dataset consisted of $N_{\text {baseline }}=47, N_{\text {CSS }}=25$, and $N_{\text {control }}=27$ scans acquired at $9.4 \mathrm{~T}$ with repetition time $=1 \mathrm{~s}$ for 360 volumes [16]. Mice were mechanically ventilated and anesthetized with $0.5 \%$ isoflurane, together with $0.05 \mathrm{mg} /$ $\mathrm{kg}$ medetomidine bolus and $0.1 \mathrm{mg} / \mathrm{kg}$ infusion (see Optogenetics: imaging for detailed protocol). The awake mouse dataset consisted of $N=54$ scans, acquired at $11.75 \mathrm{~T}$ with repetition time $=1.2 \mathrm{~s}$ for 180 volumes. The macaque dataset consisted of 10 male rhesus macaques (median age $=$ 4.98 years; median weight $=9.25 \mathrm{~kg}$ ) imaged at $3 \mathrm{~T}$ under isoflurane $(1 \%)$ anesthesia and acquired with repetition time $=2.28 \mathrm{~s}$ for 1600 volumes. The human rsfMRI dataset consisted of 15 humans imaged at $7 \mathrm{~T}$ in the awake condition with open eyes, sampled from the S1200 database [17], and was acquired with repetition time $=0.78 \mathrm{~s}$ for 900 volumes. Volume selection was performed according to two criteria: (1) activity within at least one of three seed regions-the insula, anterior cingulate area (ACA), and the dorsolateral prefrontal cortex (human), frontal eye field (macaque), or primary motor area (mouse), and (2) framewise displacement $<0.5 \mathrm{~mm}$ for macaques and humans or $0.2 \mathrm{~mm}$ for mice [22]. This led to $80.4 \%$ $(\min =73.9 \%, \max =85.3 \%)$ retained volumes on average in anesthetized mice, but $35.8 \%(\min =1,1 \%, \max =76.7 \%)$ in awake mice due to increased movement. In macaques, the percentage of retained volumes was lower $(\operatorname{mean}=19.0 \%, \min =0.1 \%, \max =53.9 \%$ ) than in humans (mean $=73.7 \%, \min =65.9 \%, \max =83.0 \%$ ). The optimal K-means clustering dimension was estimated in anesthetized mice, macaques, and humans using consensus clustering [23] from sub-samples of $80 \%$ of volumes, and the percentage of ambiguously clustered pairs [24] was used as a quality criterion. An optimum was found for $N_{\text {cluster }}=6$ in mice, $N_{\text {cluster }}$ $=5$ in macaque, and $N_{\text {cluster }}=6$ in humans. We selected $N_{\text {cluster }}=6$ across species, for comparability and in accordance with prior descriptions in anesthetized mice [25] and humans [26]. Co-activation pattern analysis operates through K-means clustering applied on the retained volumes across the whole analyzed population. Each CAP is one of the output centroids of the algorithm, that is, an average across the fMRI volumes clustered together. Since each volume is strictly associated with only one CAP, transition probabilities can be computed by quantifying how often a particular switch from the expression of a given CAP at time $t$ to that of another CAP at time $t+1$ occurs (note that if the same CAP is expressed in two consecutive frames, it counts as a transition of the CAP onto itself). The obtained normalized transition matrices, denoting this information for all possible pairs of CAPs, were vectorized and compared between species with linear models. Brain states were initially sorted between species using previously reported networks in nonhuman primates and in rodents [9-12].

\section{Synthetic brain maps}

Synthetic brain maps were generated using in situ transcriptomic similarity between available mouse in situ hybridization and human microarray data. A list of mouse and human homolog genes was obtained using Ensemble Biomart (http://www.ensembl.org) and cross-referenced to the list of genes available in the Allen Brain Atlas (https://mouse.brain-map.org/). The human data (https://human.brain-map.org/) was downloaded from the Allen Brain Atlas and processed using the automatic pipeline from the abagen ( $v$. 0.0.7) package [27]. In total, 3218 genes available in both 81 human brain regions-of-interest from the Desikan Killiany atlas [28] and mouse in situ whole-brain maps were retained. All genes were normalized to a range of $0-1$ to account for amplitude differences. Mouse brain state maps derived from the CAP analysis were modeled as a linear addition of normalized transcriptomic in situ maps using a linear model. The parameter estimates for each of the 3218 genes were applied to the human gene array and reconstructed into a 3D map overlapping with the Desikan Killiany atlas. Synthetic brain maps were compared to matched and mismatched brain states using a spatial correlation based on vectors extracted with the Desikan Killiany atlas.

\section{Projection input similarity}

Anterograde viral tracer maps $(N=498)$ obtained from injections into the right hemisphere in C57BL/6 male mice were obtained using the Allen Institute for Brain Science application programming interface and converted to NIFTI format in R using the 'nat' and 'oro.nifti' packages. Projection input similarity was estimated by extracting the tracer density profile from a seed region contralateral to the injection sites across all tracer maps and cross-referencing this profile to that of all other brain voxels. This effectively reveals regions that receive inputs from similar areas as the seed region, rather than comparing output projections from a seed region as in [29].

\section{Diffusion tensor imaging}

The S1200 7T diffusion tensor imaging dataset $(N=178)$ was reconstructed using 'bedpost $x$ ' with default parameters. Probabilistic tractography was performed using 'probtrackx', also with default parameters. Three seeds positioned on the left hemisphere were used: anterior insular area (MNI152 coordinates $-36.14,10.30,2.56)$, ACA $(-6.77,45.80,21.12)$, and dorsolateral prefrontal cortex $(-43.83,25.41,35.44)$, for the salience, default-mode, and central executive networks, respectively. Seed positions were informed from the co-activation pattern analysis. Gray-matter projections were extracted using 'fs/meants' and the Desikan Killiany atlas, and spatially compared to humanized synthetic brain maps.

\section{Optogenetics: animal permit}

All applicable international, national, and institutional guidelines for the care and use of animals were followed. All procedures performed in studies involving animals were in accordance with the ethical standards of the Institutional Animal Care and Use Committee (A*STAR Biological Resource 
Centre, Singapore, IACUC \#161134). A total of $N=18$ mice were used to acquire de novo data in this study.

\section{Optogenetics: surgery}

Mixed-sex mice ( 14-18 weeks, $\sim 25 \mathrm{~g}$ ) were anesthetized with a mixture of ketamine/xylazine (ketamine $75 \mathrm{mg} / \mathrm{kg}$, xylazine $10 \mathrm{mg} / \mathrm{kg}$ ). The head was shaved and cleaned with three wipes of Betadine and ethanol (70\%). Lidocaine was administered subcutaneously, in loco. Each animal was kept on a warm pad to maintain normal body temperature. The head was positioned in a stereotaxic frame and protective ophthalmic gel was applied to avoid dryness of the eyes. A portion of the scalp was removed to expose the skull. The skull was perforated with a drill (burr tip $\varnothing$ $\left.0.9 \mathrm{~mm}^{2}\right)$. AAV5-CaMKIla-hChR2(H134R)-mCherry $(N=10,0.75 \mu \mathrm{l}$, titer $1-8 \times 10^{12} \mathrm{vg} / \mathrm{ml}$, Vector Core at the University of North Carolina, Chapel Hill) or AAV5-CaMKIla-mCherry $(N=8)$ controls were injected at $+1.8 \mathrm{~mm}$ Anterior-Posterior, $+2.5 \mathrm{~mm}$ Medio-Lateral, $-2.0 \mathrm{~mm}$ Dorso-Ventral relative to Bregma into the left hemisphere, corresponding to the agranular insular area. A fiber-optic cannula $(\varnothing 200 \mu \mathrm{m}, 0.39 \mathrm{NA}$, length according to the injection site, $\varnothing 1.25 \mathrm{~mm}$ ceramic ferrule) was lowered to the target region (Laser 21 Pte Ltd, Singapore; Hangzhou Newdoon Technology Co., Ltd China). The cannula was fixed in place with dental cement (Meliodent rapid repair, Kulzer). Buprenorphine was administered post-surgically to each animal. Animal recovery took place on a warm pad.

\section{Optogenetics: imaging}

Animal preparation was performed as described previously [30]. Anesthesia was induced with $4 \%$ isoflurane; subsequently, the animals were endotracheally intubated, placed on an MRI-compatible cradle, and artificially ventilated (90 breaths/minute; Kent Scientific Corporation, Torrington, Connecticut, USA). A bolus with a mixture of medetomidine (Dormitor, Elanco, Greenfield, Indiana, USA) and Pancuronium Bromide (muscle relaxant, Sigma-Aldrich Pte Ltd, Singapore) was administered subcutaneously $(0.05 \mathrm{mg} / \mathrm{kg})$, followed by a maintenance infusion $(0.1 \mathrm{mg} /$ $\mathrm{kg} / \mathrm{h}$ ) while isoflurane was simultaneously reduced and kept to $0.5 \%$. Care was taken to maintain the animals' temperature at $37^{\circ} \mathrm{C}$ using a feedbackcontrolled water bath. The data were acquired on an $11.75 \mathrm{~T}$ scanner (Bruker BioSpin MRI, Ettlingen, Germany) equipped with a B-GA09S gradient system, a $72 \mathrm{~mm}$ linear volume resonator coil for transmission, and a $10 \mathrm{~mm}$ single loop surface coil. Images were acquired using Paravision 6.0.1 software. An anatomical reference scan was acquired using a spin-echo Turbo-RARE sequence: field of view $(F O V)=17 \times 9 \mathrm{~mm}^{2}$, FOV saturation slice masking non-brain regions, number of slices $=21$, slice thickness $=0.45 \mathrm{~mm}$, slice gap $=0.05 \mathrm{~mm}$, matrix dimension $(\mathrm{MD})=200 \times$ 100 , repetition time $(T R)=2742 \mathrm{~ms}$, echo time $(T E)=30 \mathrm{~ms}$, RARE factor $=$ 8 , number of averages $=2$. fMRI was acquired using a gradient-echo echoplanar imaging sequence with the same geometry as the anatomical scan: $\mathrm{MD}=60 \times 30, \mathrm{TR}=1000 \mathrm{~ms}, \mathrm{TE}=11.7 \mathrm{~ms}$, flip angle $=50^{\circ}$, volumes $=720$, bandwidth $=119047 \mathrm{~Hz}$. Field inhomogeneity was corrected using MAPSHIM protocol. ChR2 photostimulation was provided through a blue light laser (473 nm, LaserCentury, Shanghai Laser \& Optic Century Co., Ltd; $\sim 12-15 \mathrm{~mW}$ output with continuous light at the tip of the fiber). After an initial $50 \mathrm{~s}$ of rest as a baseline, $10 \mathrm{~s}$ of $20 \mathrm{~Hz} 10 \mathrm{~ms}$ light pulses were followed by $50 \mathrm{~s}$ of rest period, in a 10-block design fashion. An additional $60 \mathrm{~s}$ of rest was recorded after the last block of stimulation. Areas of photostimulation were estimated based on lesions caused by the optic fiber, as revealed with anatomical images. The photostimulation area was modeled using the default brain tissue parameters in Doric Neuroscience Studio (Doric Lenses Inc, Quebec, Canada).

\section{Literature meta-analysis}

Literature meta-analysis was performed using the neuroquery toolbox [31] for the following terms: 'depression' (https://neuroquery.org/query? text=depression + , query date: 10/07/2020) and 'positive valence' (https://neuroquery.org/query?text=positive+valence + , query date: 09/ $06 / 2020$ ). Both queries returned 78 entries, which are represented as 'overlap maps', with the default threshold applied by the neuroquery toolbox.

\section{Statistics and data representation}

Voxel-wise one-sample and two-sample $t$-tests were determined using fsl_glm, and cluster-corrected for multiple comparisons using the easythresh function, with a threshold set at either $p=0.05$ or 0.001 , as specified under the color bar. Linear models are estimated in R using the ' $I m^{\prime}$ function. Standardized coefficient parameters and 95\% confidence intervals are estimated using the 'parameters' package for R [32]. Group comparisons are represented using Gardner-Altman plots (https://www. estimationstats.com/\#) [33]. The raw data are plotted on the left or upper axes; each mean difference is plotted on the right or lower axes as a bootstrap sampling distribution. Group differences are normalized to Cohen's $d$. These are depicted as dots; 95\% confidence intervals are indicated by the ends of the vertical error bars. Inferences are made on the standardized coefficient and/or Cohen's $d$, together with their 95\% confidence intervals, following the Cohen 1988 interpretations (small: $<0.2$; medium: <0.4; large: <0.8) [34].

\section{RESULTS}

To compare resting brain activity patterns in the three species, we reduced resting-state functional Magnetic Resonance Imaging (fMRI) fluctuations acquired in human, macaque, and mouse recordings into brain states, the CAPs [21]. The human data consisted of a sub-set ( $N=15$ subjects) of the 7 Tesla Human Connectome Project S1200 dataset [17]. The macaque data ( $N=$ 10) was acquired in free-breathing anesthetized macaques [35]. The mouse data $(N=47)$ consisted of the baseline scans of ventilated mice previously acquired [16]. We clustered the images acquired in humans, macaques and mice into six brain states consisting of a spatial map and a temporal activation profile $[21,36]$. We opted for six states as this was the optimal clustering value for the mouse dataset, and was also reported as such, previously [25]. Advantageously, such a representation of brain activity is data-driven, comprehensive, and comparisons can be made in absence of exact spatial homology. We observed matching spatial patterns across species (Figs. 1a, S1). The states mainly described co-(de)activation clusters overlapping with areas previously associated with the default-mode $(\# 1,2,3)$, the salience $(\# 5,6)$, and the central executive network $(\# 1,2,4)$, referred to as the lateral cortical network in mice [9]. For instance, state \#3 displays co-activation in the anterior and posterior cingulate areas (ACA, PCA. respectively), and retro-hippocampal formation (rHPF), characteristic of the default-mode network (Fig. 1a). To account for differences between awake and anesthesia contributions, we reproduced the brain states in a dataset obtained with awake mice (Fig. S2), demonstrating brain state stability across physiological conditions. We concluded that homologous brain states exist in all three species examined.

Brain states were first matched between species based on spatial homology with previously described networks $[9,12]$. To obtain a data-driven comparison, we generated synthetic human brain states from the mouse maps. Mouse brain states were vectorized and modeled as the weighted sum of 3218 gene expression maps obtained from the in situ hybridization mouse brain database [15]. The parameter estimate for each mouse gene expression was multiplied with the homology-matched human gene expression derived from the microarray human brain database [14], and summed to obtain synthetic brain maps. Matched human brain states and corresponding synthetic maps exhibited greater spatial correlations than mismatched maps

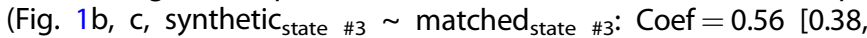
0.75], Fig. S3, matched minus mismatched: Cohen's $d=0.72$ $[-0.02,1.75])$. This confirmed our qualitative matching.

Beyond spatial homology, we sought to identify temporal homology between species. Specifically, we hypothesized that transitions between states are non-random and conserved between species. By projecting brain states into the resting-state data, we obtained state transition probabilities, as well as the number of entries into each of the six states. We found that the relative state transition probabilities between matched states were correlated between species (Fig. 1d, e, Transition ${ }_{\text {Human }} \sim$ Transition $_{\text {Mouse: }}$ Coef $=0.63[0.36,0.90]$, Transition Macaque $\sim$ Transition $_{\text {Mouse }}:$ Coef $=0.61$ 
a Human

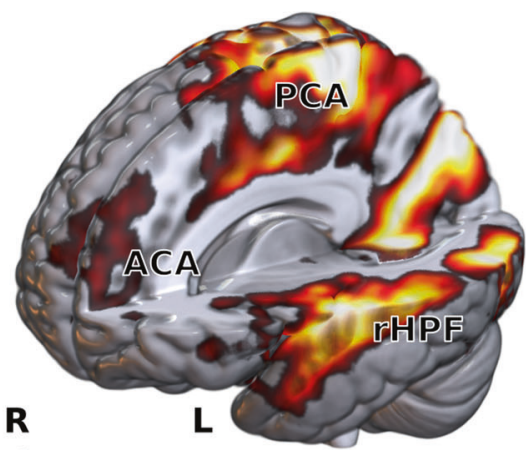

b

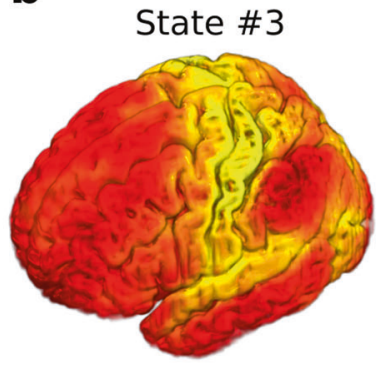

z-score

$-1.3 \quad 3.5$
Synthetic state \#3

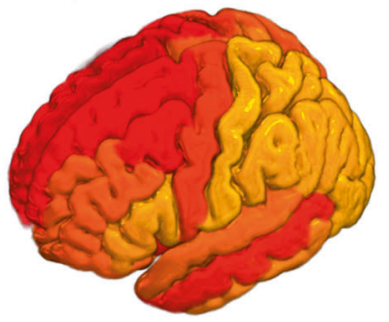

a.u.

0.2

1
Macaque

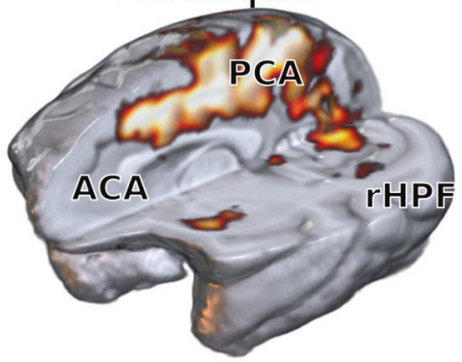

1

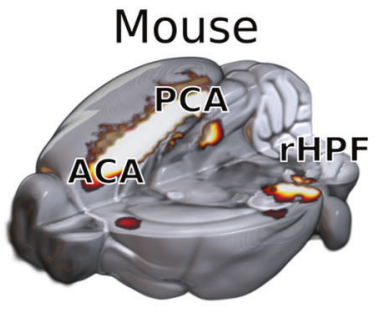

z-score

C

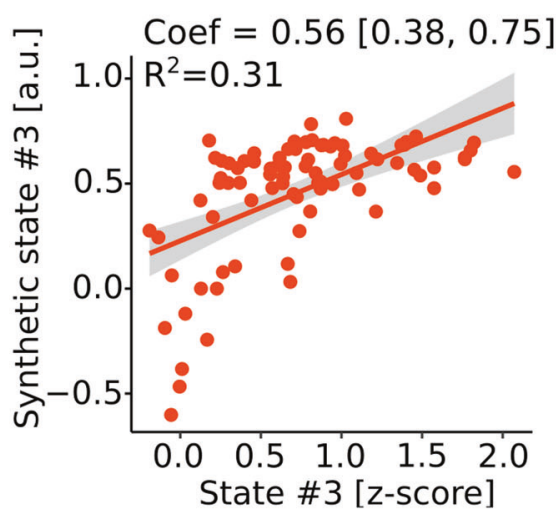

d

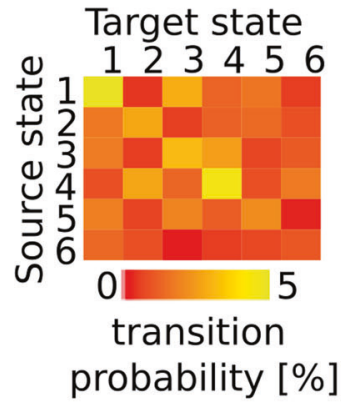

f

e
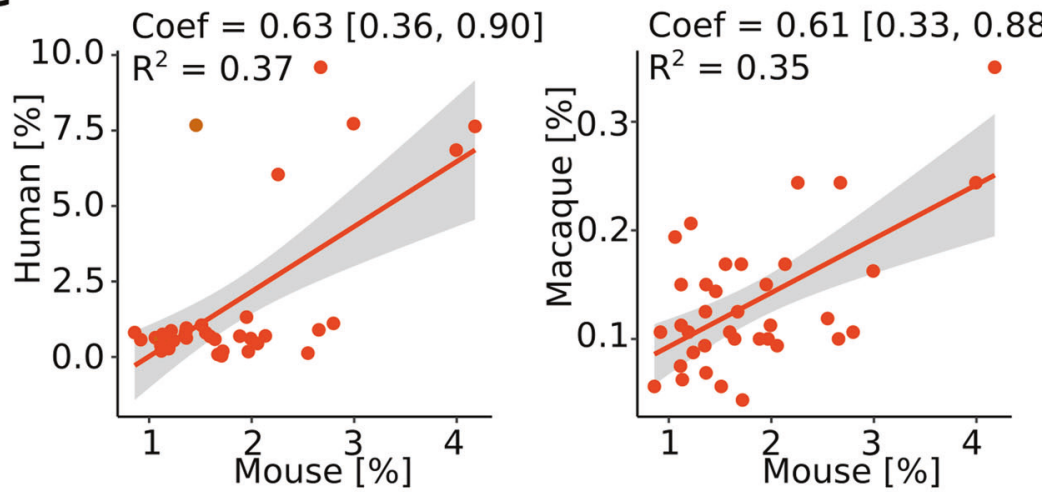

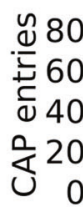
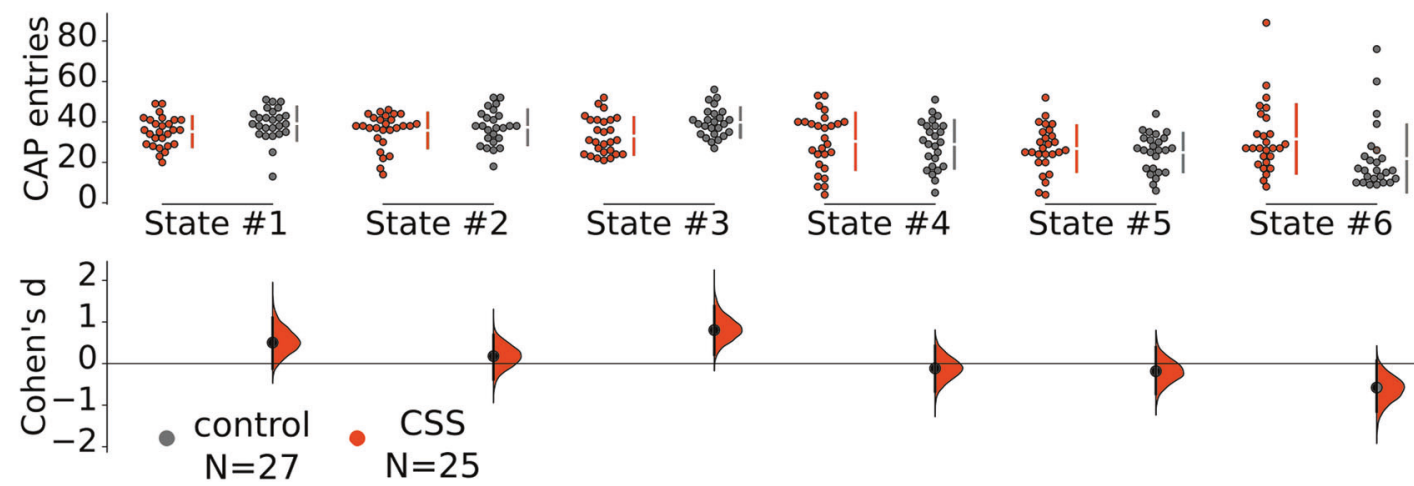

Fig. 1 Functional network homologies in three mammalian species. a Brain state \#3 exhibits default-mode-like overlap in human, macaque, and mouse brains (see Fig. S1 for details). b 3D-rendered state \#3 (left) and its synthetic map (right) obtained from the matched mouse brain state. c Spatial correlation between state \#3 and its matching synthetic map. Lines and ribbons indicate the regression lines and 95 th confidence intervals, respectively (see Fig. S3 for details). d State transition probability matrix in the mouse. e Correlated transition probabilities between the mouse and the human (left) or macaque (right). $\mathbf{f}$ Number of state entries in a chronic social stress dataset $\left(N_{\mathrm{CSS}}=\right.$

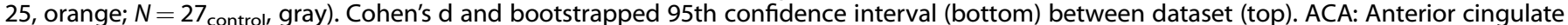
area, PCA: posterior cingulate area, rHPF: retro-hippocampal formation, CSS: chronic social stress. 

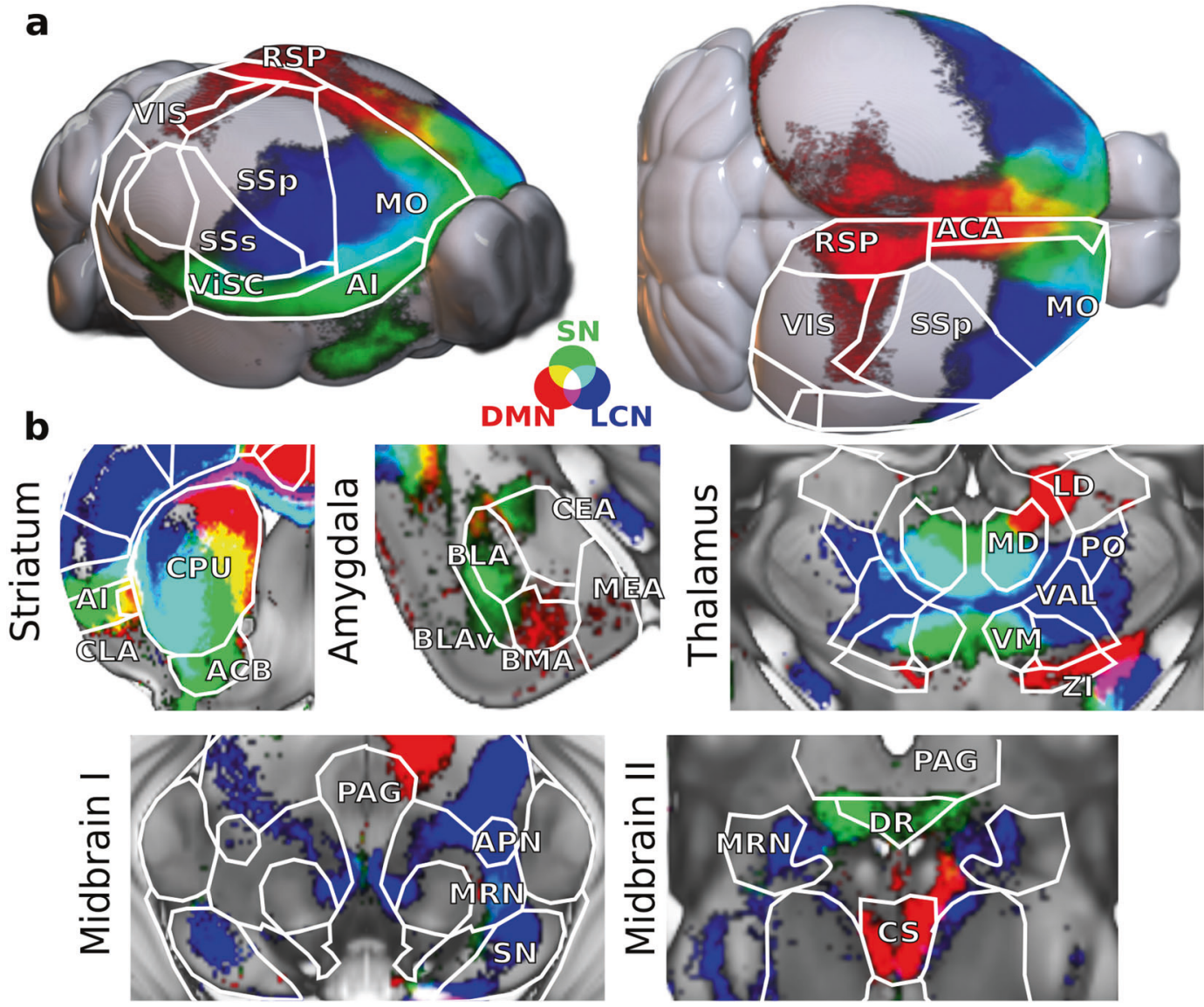

Fig. 2 A network model for the mouse. Projection input similarity in the cortex (a) and subcortical areas (b) delineate areas between the salience (SN, green), default-mode (DMN, red), or lateral cortical network (LCN, blue). Color-coded statistical maps are thresholded at $p \leq 0.05$, cluster-corrected. Extended slices are presented in Fig. S5a.

$[0.33,0.88])$. This suggests that temporal dynamics between these brain states are conserved.

Having established common spatio-temporal patterns between mice, macaques, and humans, we sought to establish if triplenetwork predictions about disease mechanisms would translate to mice. We hypothesized that, similar to humans (Fig. S4), both the default-mode and salience networks would be affected in a mouse model for depression $[16,37,38]$. We projected the brain states onto the resting-state fluctuations acquired in mice that underwent chronic social stress $\left(\right.$ CSS, $\left.N_{\text {CSS }}=25 ; \quad N_{\text {control }}=27\right)$, a paradigm to induce depression-relevant behavioral traits $[37,38]$. Consistent with our hypotheses, state \#3, overlapping with the default-mode, was entered more frequently in the stressed mice relative to controls (Fig. 1f, CSS minus control: Cohen's $d=0.80[0.21,1.38]$ ), while state \#6, overlapping with a deactivated salience network, was visited less often (Cohen's $d=$ $-0.58[-1.15,0.06])$. The brain states affected in our chronic stress model overlapped with networks implicated in depression in humans as predicted by the triple-network model and were confirmed with our meta-analysis. This similarity suggests that the mechanisms underlying brain state dysregulation in animal models of stress may translate to humans. Moreover, the presence of this phenomenon in lightly anesthetized mice suggests that this effect is hardwired rather than context-dependent.

Brain states and the functional networks that they encompass are anchored into a structural network consisting of local connections and distal projections [29, 39, 40]. Viral tracer methods remain the gold standard to establish structural networks at a high resolution [18], but are not applicable in humans. We sought to characterize the anatomical substrate underlying the network dynamics in the mouse brain. Contrary to previous endeavors that compared functional connectivity against projection outputs, we examined the projection input similarity. This framework allows for a more direct comparison to functional neuroimaging due to a common analysis using seeds. Projection input similarity for the default-mode, salience, and lateral cortical networks was determined relative to three seeds: the ACA, agranular insula (AI), and primary motor areas (MOp). This analysis showed that these three networks are anchored into chiefly non-overlapping cortical (Fig. 2a) and subcortical territories (Figs. 2b, S5a). These patterns were also recapitulated in the corresponding functional maps, consistent with the notion of structurally supported functional networks (Fig. S6). In addition to well-established cortical delineations, we generated detailed subcortical sub-divisions of the three networks (Fig. 2b). The striatum is divided across the dorsal (default-mode), lateral (lateral cortical), and ventral (salience) axes. The amygdaloid area elongates across the salience and the default-mode networks, with basolateral (BLA) and central amygdala in the former and medial (MEA) and basomedial amygdala in the latter network. The default-mode network also encompassed the zona incerta (ZI) and lateral-dorsal nucleus (LD), whereas the lateral cortical network included the ventral posterior part of the thalamus (VM). In the midbrain, the substantia nigra (SN) was associated with the lateral cortical, while the serotonin-releasing DR and the superior central raphe (CS) were part of the salience and default-mode networks, respectively. This detailed neuroanatomical model echoes similar work in humans achieved with diffusion imaging [41], albeit with enhanced detail and nucleus specificity. 

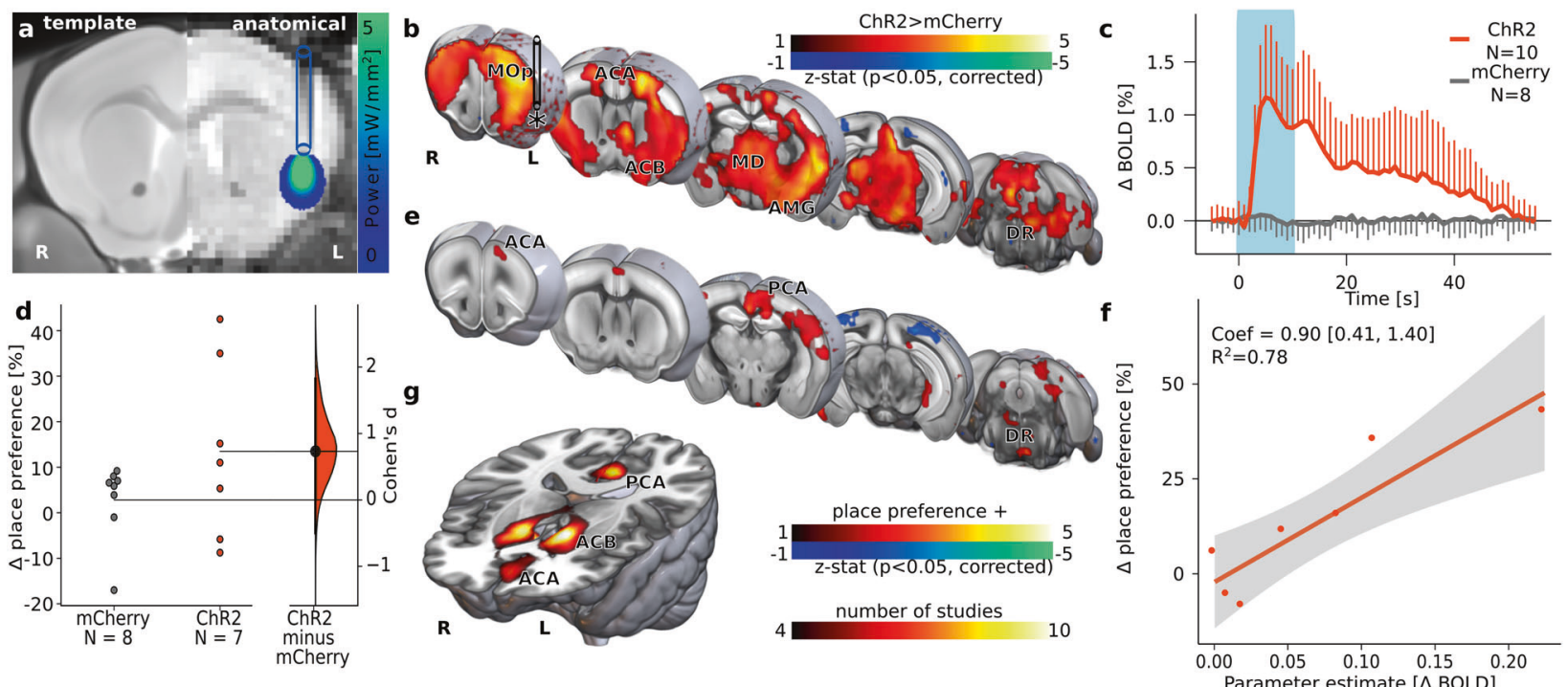

Fig. 3 Optogenetics photostimulation of the insular area. a Modeled illumination of the insula. $\mathbf{b}$ Evoked response to photostimulation blocks in ChR2 $(N=10)$ vs. mCherry $(N=8)$ controls. The asterisk indicates the photostimulation site. c Averaged insula BOLD response to photostimulation blocks. Error bars indicate \pm 1 standard deviation. d Conditioned place preference induced by photostimulation. e BOLD response correlated with place preference in ChR2-injected mice. $\mathbf{f}$ BOLD parameter estimates in the posterior cingulate area as a function of place preference. The line and ribbon indicate the regression line and 95th confidence interval, respectively. $\mathbf{g}$ Literature meta-analysis for the search term 'positive valence'.

Importantly, we observe a clear network division between the raphe nuclei (dorsal vs. central). This result suggests a revision to models for monoamine involvement in human networks that have associated the serotoninergic system with the default-mode network exclusively $[42,43]$. To validate this neuroanatomical model, we humanized the three tracer-based structural networks using the conversion method detailed above. We compared gray matter overlap with the projections estimated from probabilistic tractography in a diffusion tensor imaging dataset $(N=178)$. Both the humanized default-mode and lateral cortical network grey matter loadings were strongly associated with the tractographyestimated projections (Fig. S5b, Synthetic ${ }_{D M N} \sim$ Tractography $_{D M N}$ : Coef $=0.34[0.08,0.60]$, Synthetic $C_{\mathrm{LCN}} \sim$ Tractography $_{\text {CEN }}$ : Coef $=$ $0.60[0.27,0.94])$. The humanized mouse default-mode structural network recapitulated the key features of the human network, namely hyperintensity in the ACA, PCA, as well as medial temporal lobes (Fig. S5C). We conclude, therefore, that the mouse structural networks recapitulate features expected in humans. Further, we posit that the fine subcortical neuroanatomical details established in the mouse also translate to the human brain.

Understanding the functional contribution of these networks is central to our understanding of psychopathologies. Hence, we tested the functional integration of two nodes within the salience network implicated in depressive disorders, namely the DR nucleus and insular area. We modeled the distributed response to neuronal perturbations evoked by channelrhodopsin-2 (ChR2) photostimulation as a function of the neuroanatomical maps for the three networks (Fig. 2). First, we examined the changes in cerebral blood volume in an fMRI dataset where optogenetic (ChR2, N=63 runs) photostimulation was performed on ePetpositive DR neurons relative to yellow fluorescent protein (YFP, $N=18$ runs) controls [44]. The statistical map resulting from the comparison chiefly overlapped with that of the salience network (Fig. S7ac, Tracer $_{\mathrm{Al}} \sim$ CBV response: Coef $=0.20[0.09,0.30]$ ), consistent with the indication that the DR nucleus is embedded into the salience structural network. The second node targeted consisted of CaMKII-positive neurons in the insular area. Similar to DR ePet-positive neurons, we found that photostimulation of CaMKII-positive neurons in the insula $(N=10)$ elicited a response overlapping with the salience network relative to
mCherry-transfected controls $\left(N=8\right.$, Tracer $_{A l} \sim$ BOLD response: Coef $=0.24[0.14,0.35]$, Figs. 3a-c, S7bd, S8c). In agreement with the pivotal role of the insula in regulating activity within lateral cortical and default-mode networks [1], photostimulation of its neurons also revealed elements within these networks, respectively the primary motor areas and ACA. Given the association of the insula with the nucleus accumbens (ACB), a hub for reward, and the role of the salience network in valence mapping, we hypothesized that photostimulation of the insula would lead to positive valence, resulting in conditioned place preference. Aberrant valence mapping is associated with the induction and maintenance of depression [45]. However, we could not conclude that insula photostimulation leads to positive valence (Fig. 3d, ChR2 minus mCherry: Cohen's $d=0.73[-0.49,1.83])$. The variability in the evoked behavior in the ChR2 group could be due to individual differences in network engagement to photostimulation. To test this, we regressed the place preference parameter into individual functional maps. This revealed clusters in the ACA, PCA, both positively associated with place preference (Fig. $3 e, f, \triangle$ place preference $\sim$ PCA: Coef $=0.90[0.41,1.40])$. To test how cingulate involvement in positive valence translates to humans, the optogenetic response was compared to a meta-analysis for the search term 'positive valence' in human neuroimaging studies (Fig. 3g). There was an elevated overlap in the literature in association with 'positive valence' in both ACA, PCA. These loci are also highly associated with the default-mode network and they are areas highlighted in our 'depression' meta-analysis (Fig. S4). We conclude that specific network engagement between the salience and default-mode networks enhances the conditioning for place preference.

\section{DISCUSSION}

We demonstrate that resting-state fluctuations consist of dynamically occurring brain states that are conserved across three species, and encode pathological manifestations, such as depression-relevant conditions in mice. Further, we demonstrate a triple-network system in mice and the detailed anatomical subcortical substrate that supports it. Finally, we show that evoked activity within the nodes belonging to the salience network preferentially elicits activity within that network and that 
photostimulation of the insula elicits a positive valence when coactivation of cingulate areas is present. Taken together, we provide a model highlighting neuromodulation targets to control specific networks and to serve as a common currency to translate findings between neurobiological observations in rodents and network dynamics assessed in humans with neuroimaging.

We demonstrate the existence of homologous brain states in the human, macaque, and mouse brains. Unexpectedly, we find that the serotoninergic system is associated with different elements of the triple network; specifically, the DR was found to be structurally and functionally associated with the salience network. This contrasts with previous work in humans that has suggested an association with the default-mode network [42, 43]. Our observation is further supported by other studies in rodents that have shown the involvement of the ventral striatum, a node of the salience network, in response to psilocybin, a 5HT2a receptor agonist [46, 47]. Puzzlingly, this contrasts with a comparable study in humans that indicated default-mode network involvement instead [48] (but also a ventral striatum response [49]). Whether these network involvement inconsistencies are due to species differences, or to anesthesia, will need to be determined; however, viral tracers are independent of anesthesia, providing anatomical support for our hypothesis. Finally, it should be noted that the serotoninergic system extends beyond the dorsal and CS nuclei, with at least nine nuclei characterized and each containing heterogeneous neuronal populations [50]. The regulation of distributed neuronal networks is expected to be mediated by complex mechanisms.

This work is facilitated by the availability of large online databases for human and mouse rsfMRI, meta-analysis tools, diffusion imaging, viral tracers, and spatial transcriptomics $[14,15,17,18,27,31]$. While we used nonhuman primate data as an intermediary for our first comparison, the relative lack of resources, such as spatial transcriptomics, highlights a need in the community for additional nonhuman primate resources to strengthen our comparisons [51, 52]. Fortunately, publicly available nonhuman primate resources are growing; this will allow for future comparisons across different modalities and scales between species, to better unravel the puzzling mechanisms leading to abnormal neuronal network function.

\section{REFERENCES}

1. Menon V. Large-scale brain networks and psychopathology: a unifying triple network model. Trends Cogn Sci. 2011;15:483-506.

2. Whitfield-Gabrieli S, Ford JM. Default mode network activity and connectivity in psychopathology. Annu Rev Clin Psychol. 2012;8:49-76.

3. Sprooten E, Rasgon A, Goodman M, Carlin A, Leibu E, Lee WH, et al. Addressing reverse inference in psychiatric neuroimaging: Meta-analyses of task-related brain activation in common mental disorders. Hum Brain Mapp. 2017;38:1846-64.

4. Goodkind M, Eickhoff SB, Oathes DJ, Jiang Y, Chang A, Jones-Hagata LB, et al. Identification of a common neurobiological substrate for mental illness. JAMA Psychiatry. 2015;72:305-15.

5. Janiri D, Moser DA, Doucet GE, Luber MJ, Rasgon A, Lee WH, et al. Shared Neural Phenotypes for Mood and Anxiety Disorders: a Meta-analysis of 226 Task-Related Functional Imaging Studies. JAMA Psychiatry. 2020;77:172-9.

6. Uddin LQ, Kelly AM, Biswal BB, Castellanos FX, Milham MP. Functional connectivity of default mode network components: correlation, anticorrelation, and causality. Hum Brain Mapp. 2009;30:625-37.

7. Fox MD, Snyder AZ, Vincent JL, Corbetta M, Van Essen DC, Raichle ME. The human brain is intrinsically organized into dynamic, anticorrelated functional networks. Proc Natl Acad Sci USA. 2005;102:9673-8.

8. Damoiseaux JS, Rombouts SARB, Barkhof F, Scheltens P, Stam CJ, Smith SM. et al. Consistent resting-state networks across healthy subjects.Proc Natl Acad Sci USA. 2006;103:13848-53.

9. Gozzi A, Schwarz AJ. Large-scale functional connectivity networks in the rodent brain. Neuroimage 2016;127:496-509.

10. Lu H, Zou Q, Gu H, Raichle ME, Stein EA, Yang Y. Rat brains also have a default mode network. Proc Natl Acad Sci USA. 2012;109:3979-84.
11. Tsai P-J, Keeley RJ, Carmack SA, Vendruscolo JCM, Lu H, Gu H, et al. Converging Structural and Functional Evidence for a Rat Salience Network. Biol Psychiatry. 2020;88:867-78.

12. Hutchison RM, Everling S. Monkey in the middle: why non-human primates are needed to bridge the gap in resting-state investigations. Front Neuroanat. 2012;6:29.

13. van Heukelum S, Mars RB, Guthrie M, Buitelaar JK, Beckmann CF, Tiesinga PHE, et al. Where is Cingulate Cortex? A Cross-Species View. Trends Neurosci. 2020;43:285-99.

14. Hawrylycz MJ, Lein ES, Guillozet-Bongaarts AL, Shen EH, Ng L, Miller JA, et al. An anatomically comprehensive atlas of the adult human brain transcriptome. Nature. 2012;489:391-9.

15. Lein ES, Hawrylycz MJ, Ao N, Ayres M, Bensinger A, Bernard A, et al. Genome-wide atlas of gene expression in the adult mouse brain. Nature. 2007;445:168-76.

16. Grandjean J, Azzinnari D, Seuwen A, Sigrist H, Seifritz E, Pryce CR, et al. Chronic psychosocial stress in mice leads to changes in brain functional connectivity and metabolite levels comparable to human depression. Neuroimage. 2016;142:544-52.

17. Van Essen DC, Smith SM, Barch DM, Behrens TEJ, Yacoub E, Ugurbil K, et al. The WU-Minn Human Connectome Project: an overview. Neuroimage. 2013;80:62-79.

18. Oh SW, Harris JA, Ng L, Winslow B, Cain N, Mihalas S, et al. A mesoscale connectome of the mouse brain. Nature 2014;508:207-14.

19. Felleman DJ, Van Essen DC. Distributed Hierarchical Processing in the Primate Cerebral Cortex. Cereb Cortex. 1991;1:1-47.

20. Avants BB, Tustison NJ, Stauffer M, Song G, Wu B, Gee JC. The Insight ToolKit image registration framework. Front Neuroinform. 2014;8:44.

21. Bolton TAW, Tuleasca C, Wotruba D, Rey G, Dhanis H, Gauthier B, et al. TbCAPs: a toolbox for co-activation pattern analysis. Neuroimage. 2020;211:116621.

22. Power JD, Barnes KA, Snyder AZ, Schlaggar BL, Petersen SE. Spurious but systematic correlations in functional connectivity MRI networks arise from subject motion. Neuroimage. 2012;59:2142-54.

23. Monti S. Machine Learning. 2003;52:91-18. https://doi.org/10.1023/ A:1023949509487.

24. Șenbabaoğlu Y, Michailidis G, Li JZ. Critical limitations of consensus clustering in class discovery. Sci Rep. 2014;4:6207.

25. Gutierrez-Barragan D, Basson MA, Panzeri S, Gozzi A. Infraslow State Fluctuations Govern Spontaneous fMRI Network Dynamics. Curr Biol. 2019; 29:2295-306.e5.

26. Bolton TAW, Wotruba D, Buechler R, Theodoridou A, Michels L, Kollias $S$, et al. Triple Network Model Dynamically Revisited: Lower Salience Network State Switching in Pre-psychosis. Front Physiol. 2020;11:66.

27. Markello R, Shafiei $G$, Zheng $Y-Q$, Mišić $B$ abagen: A toolbox for the Allen Brain Atlas genetics data. 2020. https://doi.org/10.5281/zenodo.3688800.

28. Desikan RS, Ségonne F, Fischl B, Quinn BT, Dickerson BC, Blacker D, et al. An automated labeling system for subdividing the human cerebral cortex on MRI scans into gyral based regions of interest. Neuroimage. 2006:31:968-80.

29. Grandjean J, Zerbi V, Balsters JH, Wenderoth N, Rudin M. Structural Basis of LargeScale Functional Connectivity in the Mouse. J Neurosci. 2017;37:8092-101.

30. Grandjean J, Schroeter A, Batata I, Rudin M. Optimization of anesthesia protocol for resting-state $\mathrm{fMRI}$ in mice based on differential effects of anesthetics on functional connectivity patterns. Neuroimage. 2014;102:838-47. Pt 2

31. Dockès J, Poldrack RA, Primet R, Gözükan H, Yarkoni T, Suchanek F, et al. NeuroQuery, comprehensive meta-analysis of human brain mapping. Elife. 2020;9: e53385.

32. Lüdecke D, Ben-Shachar M, Patil I, Makowski D. Extracting, computing and exploring the parameters of statistical models using R. J Open Source Softw. 2020;5:2445.

33. Ho J, Tumkaya T, Aryal S, Choi H, Claridge-Chang A. Moving beyond P values: data analysis with estimation graphics. Nat Methods. 2019;16:565-6.

34. Cohen J. Statistical Power. 2nd ed. Mahwah, USA: Lawrence Erlbaum Associates; 1988.

35. Balsters JH, Zerbi V, Sallet J, Wenderoth N, Mars RB. Primate homologs of mouse cortico-striatal circuits. Elife. 2020;9:e53680.

36. Preti MG, Bolton TA, Van De Ville D. The dynamic functional connectome: stateof-the-art and perspectives. Neuroimage. 2017;160:41-54.

37. Azzinnari D, Sigrist H, Staehli S, Palme R, Hildebrandt T, Leparc G, et al. Mouse social stress induces increased fear conditioning, helplessness and fatigue to physical challenge together with markers of altered immune and dopamine function. Neuropharmacology. 2014;85:328-41.

38. Golden SA, Covington HE III, Berton O, Russo SJ. A standardized protocol for repeated social defeat stress in mice. Nat Protoc. 2011;6:1183-91.

39. Honey CJ, Sporns O, Cammoun L, Gigandet X, Thiran JP, Meuli R, et al. Predicting human resting-state functional connectivity from structural connectivity. Proc Natl Acad Sci USA. 2009;106:2035-40. 
40. Stafford JM, Jarrett BR, Miranda-Dominguez O, Mills BD, Cain N, Mihalas S, et al. Large-scale topology and the default mode network in the mouse connectome. Proc Natl Acad Sci USA. 2014;111:18745-50.

41. Behrens TEJ, Johansen-Berg $H$, Woolrich MW, Smith SM, Wheeler-Kingshott CAM, Boulby PA, et al. Non-invasive mapping of connections between human thalamus and cortex using diffusion imaging. Nat Neurosci. 2003;6:750-7.

42. Conio B, Martino M, Magioncalda $P$, Escelsior A, Inglese $M$, Amore $M$, et al. Opposite effects of dopamine and serotonin on resting-state networks: review and implications for psychiatric disorders. Mol Psychiatry. 2020;25:82-93.

43. Bär K-J, de la Cruz F, Schumann A, Koehler S, Sauer H, Critchley H, et al. Functional connectivity and network analysis of midbrain and brainstem nuclei. Neuroimage. 2016;134:53-63.

44. Grandjean J, Corcoba A, Kahn MC, Upton AL, Deneris ES, Seifritz E, et al. A brainwide functional map of the serotonergic responses to acute stress and fluoxetine. Nat Commun. 2019;10:350.

45. Medeiros GC, Rush AJ, Jha M, Carmody T, Furman JL, Czysz AH, et al. Positive and negative valence systems in major depression have distinct clinical features, response to antidepressants, and relationships with immunomarkers. Depress Anxiety. 2020;37:771-83.

46. Grandjean J, Buehlmann D, Buerge M, Sigrist H, Seifritz E, Vollenweider FX, et al. Psilocybin exerts distinct effects on resting state networks associated with serotonin and dopamine in mice. Neuroimage. 2021;225:117456.

47. Sakashita Y, Abe K, Katagiri N, Kambe T, Saitoh T, Utsunomiya I, et al. Effect of psilocin on extracellular dopamine and serotonin levels in the mesoaccumbens and mesocortical pathway in awake rats. Biol Pharm Bull. 2015;38:134-8.

48. Carhart-Harris RL, Erritzoe D, Williams T, Stone JM, Reed LJ, Colasanti A, et al. Neural correlates of the psychedelic state as determined by fMRI studies with psilocybin. Proc Natl Acad Sci USA. 2012;109:2138-43.

49. Vollenweider F. 5-HT Modulation of Dopamine Release in Basal Ganglia in Psilocybin-Induced Psychosis in Man-A PET Study with [11C]raclopride. Neuropsychopharmacology. 1999;20:424-33.

50. Huang KW, Ochandarena NE, Philson AC, Hyun M, Birnbaum JE, Cicconet $M$, et al. Molecular and anatomical organization of the dorsal raphe nucleus. 2019. https:// doi.org/10.7554/eLife.46464.

51. Milham MP, Ai L, Koo B, Xu T, Amiez C, Balezeau F, et al. An Open Resource for Non-human Primate Imaging. Neuron. 2018;100:61-74.e2.

52. Yin S, Lu K, Tan T, Tang J, Wei J, Liu X, et al. Transcriptomic and open chromatin atlas of high-resolution anatomical regions in the rhesus macaque brain. Nat Commun. 2020;11:474

\section{ACKNOWLEDGMENTS}

This work was supported by the Singapore Biolmaging Consortium core funding and the Singapore Biolmaging Consortium award \#2016 to JGr and \#2017 to FM. FM was supported by a Ph.D. scholarship funded through the A*STAR Research Attachment Programme and the University of Manchester (awarded to JGi). RMV was supported by the Dutch Research Council grant OCENW.KLEIN.334 (awarded to JGr). This work was supported by the A*STAR Investigatorship (awarded to FY). RBM is supported by the Biotechnology and Biological Science Research Council (BBSRC) [BB/N019814/1]. The Wellcome Centre for Integrative Neuroimaging is supported by core funding from the Wellcome Trust [203129/Z/16/Z]. Data were provided in part by the Human Connectome Project, WU-Minn Consortium (Principal Investigators: David Van Essen and Kamil Ugurbil; 1U54MH091657) funded by the $16 \mathrm{NIH}$ Institutes and Centers that support the NIH Blueprint for Neuroscience Research; and by the McDonnell Center for Systems Neuroscience at Washington University. The authors thank Profs CR Pryce and D van de Ville and Dr Corey Horien for critically reading the paper.

\section{AUTHOR CONTRIBUTIONS}

FM: Conceptualization, Methodology, Data acquisition. RMV: Conceptualization, Methodology. HEF: Methodology, Data acquisition. LYY: Methodology, Data acquisition. TAWB: Methodology, Software. PS: Conceptualization. CLT: Data acquisition. CYL: Data acquisition. AB: Conceptualization. SL: Methodology. RB: Methodology. JZ: Data acquisition. GHTL: Data acquisition. NL: Data acquisition. JS: Resources. JG: Resources, Writing-Review \& Editing, Supervision, Funding acquisition. JPL: Conceptualization. RBM: Conceptualization, Resources, Writing-Review \& Editing. OM: Resources, Writing - Review \& Editing, Supervision, Funding acquisition. YF: Resources, WritingReview \& Editing, Supervision, Funding acquisition. JG: Conceptualization, Methodology, Data acquisition, Formal analysis, Data curation, Software, Resources, WritingOriginal Draft, Writing-Review \& Editing, Supervision, Funding acquisition.

\section{COMPETING INTERESTS}

JGr receives funding as a consultant for Novartis International AG. The latter was not involved in the design, analysis, or reporting of this study. The other authors have no competing interests to disclose.

\section{ADDITIONAL INFORMATION}

Supplementary information The online version contains supplementary material available at https://doi.org/10.1038/s41380-021-01298-5.

Correspondence and requests for materials should be addressed to Joanes Grandjean.

Reprints and permission information is available at http://www.nature.com/ reprints

Publisher's note Springer Nature remains neutral with regard to jurisdictional claims in published maps and institutional affiliations.

\section{cc) (i)}

Open Access This article is licensed under a Creative Commons Attribution 4.0 International License, which permits use, sharing, adaptation, distribution and reproduction in any medium or format, as long as you give appropriate credit to the original author(s) and the source, provide a link to the Creative Commons license, and indicate if changes were made. The images or other third party material in this article are included in the article's Creative Commons license, unless indicated otherwise in a credit line to the material. If material is not included in the article's Creative Commons license and your intended use is not permitted by statutory regulation or exceeds the permitted use, you will need to obtain permission directly from the copyright holder. To view a copy of this license, visit http://creativecommons. org/licenses/by/4.0/.

(c) The Author(s) 2021 